Musées, Patrimoine et Culture scientifiques et techniques

$119 \mid 2008$

septembre - octobre 2008

\title{
Leuglay : un centre d'interprétation de la forêt et des métiers du bois
}

\section{Serge Chaumier}

\section{OpenEdition \\ Journals}

Édition électronique

URL : http://journals.openedition.org/ocim/356

DOI : 10.4000/ocim.356

ISSN : 2108-646X

Éditeur

OCIM

Édition imprimée

Date de publication : 1 septembre 2008

Pagination : 26-30

ISSN : 0994-1908

Référence électronique

Serge Chaumier, «Leuglay : un centre d'interprétation de la forêt et des métiers du bois », La Lettre de I'OCIM [En ligne], 119 | 2008, mis en ligne le 21 janvier 2011, consulté le 19 avril 2019. URL : http:// journals.openedition.org/ocim/356 ; DOI : 10.4000/ocim.356 
en investissement, a reçu le soutien financier de la Région Bourgogne complété de crédits départementaux et européens. La conduite des opérations a été coordonnée par Agnès Levillain, muséographe missionnée par le centre de Recherche sur la Culture et les Musées de l'université de Bourgogne, avec la contribution de Jean-Marc Gaillard pour la scénographie et de Marc Perrey pour les réalisations audiovisuelles. Le site, soutenu par les collectivités territoriales, est géré à présent par une association.

\section{Un lieu pour susciter la rencontre des regards}

Le centre d'interprétation met en valeur les aspects naturels et environnementaux, les domaines de loisirs qui y sont liés, mais aussi les aspects professionnels de la gestion de la forêt. Une pluralité de regards est donc proposée, dans un traitement anthropologique du sujet. L'idée est de croiser les approches afin de sensibiliser chacun à des usages différenciés et à des logiques qui demeurent parfois étrangères les unes aux autres ou qui ont des difficultés à dialoguer. Celle de l'environnementaliste, du chasseur, de l'office national de gestion des forêts, des forestiers et de ceux qui développent une activité économique, des riverains qui entendent se chauffer au bois ou encore celle des ramasseurs de champignons ou des sportifs qui l'utilisent comme terrain d'exploration et d'exercice : autant de sensibilités qui considèrent différemment un même espace.

L'histoire de la forêt, de l'arbre et son fonctionnement, des écosystèmes forestiers, de l'industrie du bois, sont des passages obligés du propos de l'exposition, qui se prolongent par des dimensions techniques, environnementalistes, mais aussi métaphoriques. Les contes et légendes liés à la forêt, et les imaginaires véhiculés, la façon dont les populations ont développé avec elle des relations particulières dans l'histoire, les prévisions que l'on peut avoir de son rôle dans l'avenir, sont autant de pistes de réflexion que l'exposition suit pour éveiller la curiosité et l'étonnement du visiteur. Les métiers de la forêt et du bois sont variés et souvent mal connus. Pour en rendre compte, des séquences vidéos sont proposées sur différents secteurs d'activités, par exemple la production du charbon de bois. Le visiteur va du bûcheronnage traditionnel à ses évolutions modernes, la scierie ou encore les convoyages des billes de bois...

L'objet tient une place et un rôle autres que dans un musée « traditionnel ». S'il y a quelques objets mobilisés, demeure central le discours développé pour guider le visiteur dans une découverte des aspects qu'il connaît mal ou qu'il ne suspecte pas. Certes, la botanique et les espèces animales sont des dimensions qui permettent de se familiariser à un écosystème, mais ce sont les aspects moins prévisibles qui sont surtout privilégiés. Spécificité de la forêt bourguignonne en regard des autres forêts nationales et internationales, évolution dans le temps, et mode de gestion des espaces amènent à surprendre le visiteur souvent enclin à estimer la surface de forêt en recul. De même, des idées préconçues sur la consommation de papier et sur l'économie du bois sont discutées pour dévoiler des aspects souvent surprenants pour le citadin : par exemple qu'il est utile de consommer du bois pour entretenir les forêts...

Le centre d'interprétation s'efforce de répondre à sa définition initiale, à savoir de mettre en perspective des avis contradictoires pour amener le visiteur à réfléchir par lui-même à partir d'éléments objectifs ; et à construire ainsi sa propre opinion. Certes, dans la réalité, les commanditaires ont souvent des difficultés à accepter de telles approches, et les avis contradictoires demeurent encore timides au regard de ce qui pourrait être fait. "Le propre de l'interprétation est de stimuler chez le visiteur un désir d'élargir l'horizon de ses intérêts et de ses connaissances et de l'aider à comprendre les grandes vérités qui gisent derrière tout constat des faits », écrit Tilden, le père de l'interprétation. Dans cette veine, la maison de la Forêt apporte des éléments d'information et d'appréciation pour que le visiteur puisse prolonger ensuite ses perceptions, par la confrontation directe, en forêt ou dans sa vie quotidienne.

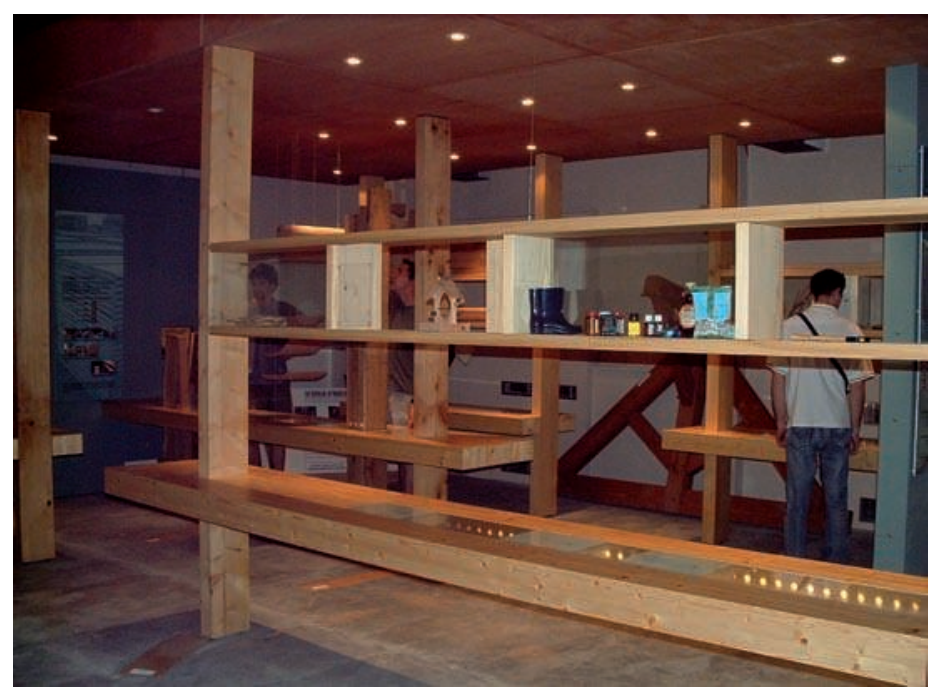

Exposition de sensibilisation au milieu forestier (c) Maison de la Forêt 


\section{Les outils mobilisés}

Plusieurs types de médiation sont utilisés pour donner à la visite une approche dynamique et recourir aux techniques les plus efficaces selon le sujet traité. Des audiovisuels proposent des courts reportages, ainsi cinq bornes multimédia présentent près de $36 \mathrm{mi}$ nutes de films séquencés en petites unités de quelques minutes. Des manips, des dispositifs sonores et olfactifs, des textes et de l'iconographie bien évidemment, multiplient les approches pour proposer un panorama le plus complet possible. L'exposition permanente s'appuie principalement sur l'expérience sensible. Toucher, sentir, entendre, rêver, imaginer sont les principales voies d'accès à la compréhension pour le visiteur. La volonté pédagogique du centre d'interprétation est indéniable : elle se place au cœur des contenus de l'exposition, des programmes d'actions et d'animation. À partir d'une assise scientifique rigoureuse, elle explore les potentialités par des approches combinées et variées. Bien sûr la médiation humaine des animateurs demeure centrale et indispensable, notamment pour les groupes.

"Processus de communication qui vise à transmettre au visiteur la signification et la valeur d'aspects privilégiés du patrimoine culturel et naturel aux moyens d'expériences sensibles avec des objets, des artefacts, des paysages ou des sites ", cette préconisation de Tilden est mise en action à la maison de la Forêt par divers moyens. L'espace d'exposition de $450 \mathrm{~m}^{2}$ demeure modeste, mais c'est aussi par les activités connexes que le site prend toute son ampleur grâce à un système de propositions en complémentarité. Ainsi un arboretum sur la partie extérieure, des sentiers de découverte en forêt, des ateliers pédagogiques, une partie laboratoire, une salle multimédia, de petites expositions temporaires, des journées thématiques (champignons...), des activités sportives (la « grimpe d'arbres » ou Accrobranches) complètent le dispositif. Des parcours GPS ont nouvellement été mis en place pour allier cette nouvelle technologie à une exploration forestière. La visite de la chaudière à particules bois, financée avec l'ADEME pour équiper le bâtiment réhabilité, prend tout son sens pour traiter, par l'exemple, des économies d'énergie et de la place que peut prendre la filière bois dans les modes énergétiques. Il faut signaler enfin un fonds documentaire conséquent, la bibliothèque Georges Plaisance, nom de cet ingénieur forestier qui fait autorité en la matière, fonds qui constitue un outil de ressources pour les chercheurs sur le thème de la forêt.

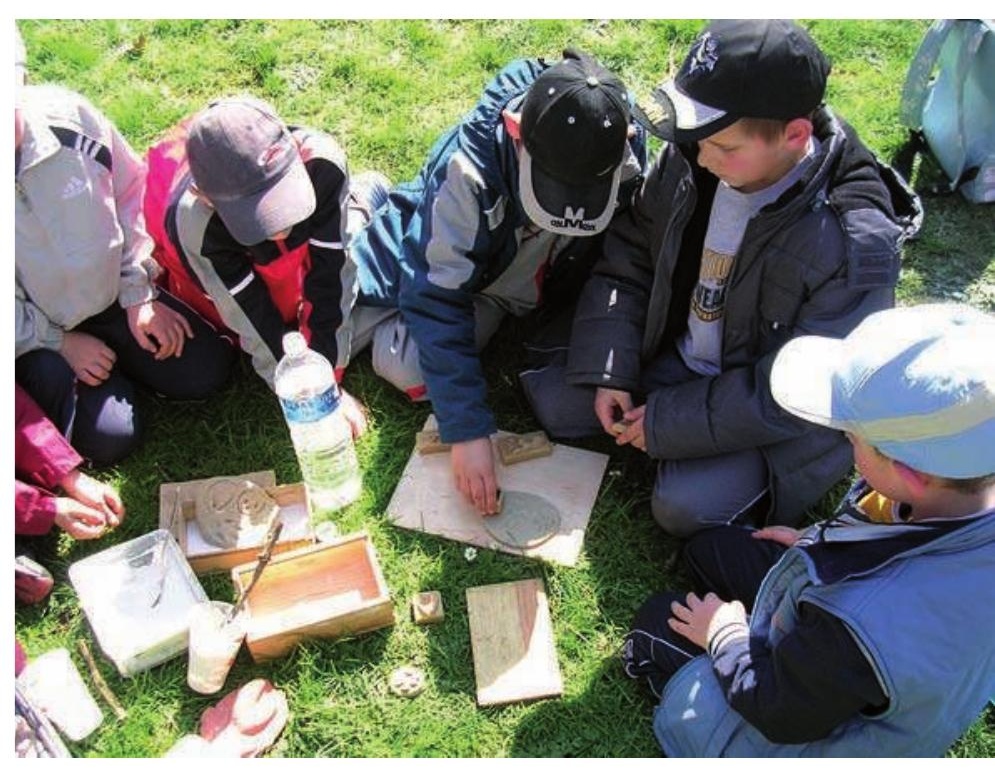

Activités de moulage des empreintes en forêt avec un groupe scolaire (c) Maison de la Forêt

Mais le panorama serait incomplet si la maison de la Forêt n'avait pas cherché à valoriser également d'autres modes de perception de la forêt, notamment plus poétiques, littéraires ou artistiques. Ainsi des œuvres sont intégrées dans le parcours d'exposition pour apporter des visions subjectives et suggestives sur la forêt et ses imaginaires. De grands noms de l'art contemporain se sont vus commander des installations alors que d'autres œuvres ont pu être mises en dépôt par le FRAC Bourgogne. C'est le cas d'une vision de la forêt, signée Bertrand Lavier, compagnon de route de ce territoire du Châtillonnais. La Fondation de Bourgogne a supervisé les commandes passées à des artistes, notamment à Marc Mimram pour une passerelle d'accès au site, Jacques Vieille propose une construction en chutes de parquet et une architecture végétale, Laetitia de Bazelaire une volière et Jacques Simon a réalisé des œuvres en salle et des aménagements extérieurs et paysagers. On peut regretter que ce patrimoine artistique constitutif ne soit pas enrichi progressivement et que les acteurs locaux semblent finalement se désintéresser d'un apport qui permet d'ouvrir la thématique et le territoire en renouvelant ses approches.

\section{Un projet de développement local}

L'ancrage de la maison de la Forêt au sein de la communauté du Pays Châtillonnais est un exemple de ce qu'un centre d'interprétation peut offrir à un public local comme à un public extérieur pour créer 
une synergie d'intérêts. Le revers de la médaille est que cette région de forêt, donc peu habitée, peu touristique, est par conséquent peu passagère. Dans cette situation, un lieu d'exposition est une façon d'inverser quelque peu la situation en développant des offres attractives, mais bien évidemment avec les difficultés inhérentes à ce type de développement encore balbutiant. Dans ce sens, la maison de la Forêt a été dimensionnée pour un fonctionnement correspondant à des perspectives raisonnables de développement touristique de la région. Ses coûts de fonctionnement sont limités, son espace est adapté à une juste fréquentation. Si le lieu connaît des périodes difficiles, ce qui n'est pas surprenant, c'est aussi en maintenant un engagement volontaire par l'investissement dans ce type d'équipement que les pouvoirs publics pourront impulser le développement territorial. Un projet de réhabilitation de l'ancienne gare de Leuglay en structure d'accueil permettra sans doute d'offrir des possibilités accrues à l'avenir pour le tourisme.

Le centre d'interprétation a pour vocation d'accueillir tout type de public, mais, l'accent a été mis avant tout sur les publics scolaires. Ils représentent plus de $60 \%$ des visiteurs du centre. Ceux-ci ont l'habitude de se tourner vers les structures à vocation culturelle pour conduire des projets pédagogiques « hors les murs » et chercher de nouveaux terrains d'investigation des savoirs. Visites, activités sportives ou scientifiques, centre de ressources, la maison de la Forêt offre un panel de possibilités que recherchent les enseignants et les responsables de centres de vacances. L'espace d'exposition constitue en ce sens une porte d'entrée sur le territoire forestier et sur les explorations multiples qu'il propose.

D'un point de vue scientifique, il a été décidé lors de l'élaboration des contenus de l'exposition que les données socio-économiques, historiques, géographiques et écologiques utilisées proviendraient du Pays Châtillonnais dans son ensemble, unité géographique retenue pour le propos. Le centre d'interprétation porte par conséquent sur un territoire plus large que la seule commune de Leuglay et les visiteurs sont invités à découvrir l'ensemble du Pays Châtillonnais dès leurs premiers pas dans l'exposition (carte du Pays Châtillonnais au début de l'exposition). Ceci a pour effet d'accroître le discours et d'en faire un lieu de ressource plus global pour l'ensemble du bassin de vie, raison qui conduit la communauté de communes a être le niveau institutionnel d'implication adéquat.

\section{Une gestion partagée, des partenariats démultipliés}

Un équipement tel que celui de la maison de la Forêt nécessite un mode de gestion commun où l'ensemble des acteurs locaux (issus des entreprises de la filière bois, des associations sportives et culturelles, des au tres lieux à vocation touristique, du monde des artistes, du monde politique...) se fédère autour de ce projet pour le faire vivre. La maison de la Forêt est un lieu de vie sociale, culturelle, économique et touristique du territoire. Dans ce sens, il est intéressant de noter que le lieu sert dans le cadre de la formation professionnelle des jeunes dont la vocation se tourne vers les métiers de la filière bois, des partenariats avec les lycées professionnels sont ainsi privilégiés. Le centre d'interprétation de la Forêt de Leuglay organise des séjours forestiers spécifiques pour les BTS Gestion Forestière ou les BTS Technico Commerciaux. Des visites et des stages de formation s'y déroulent, et plus

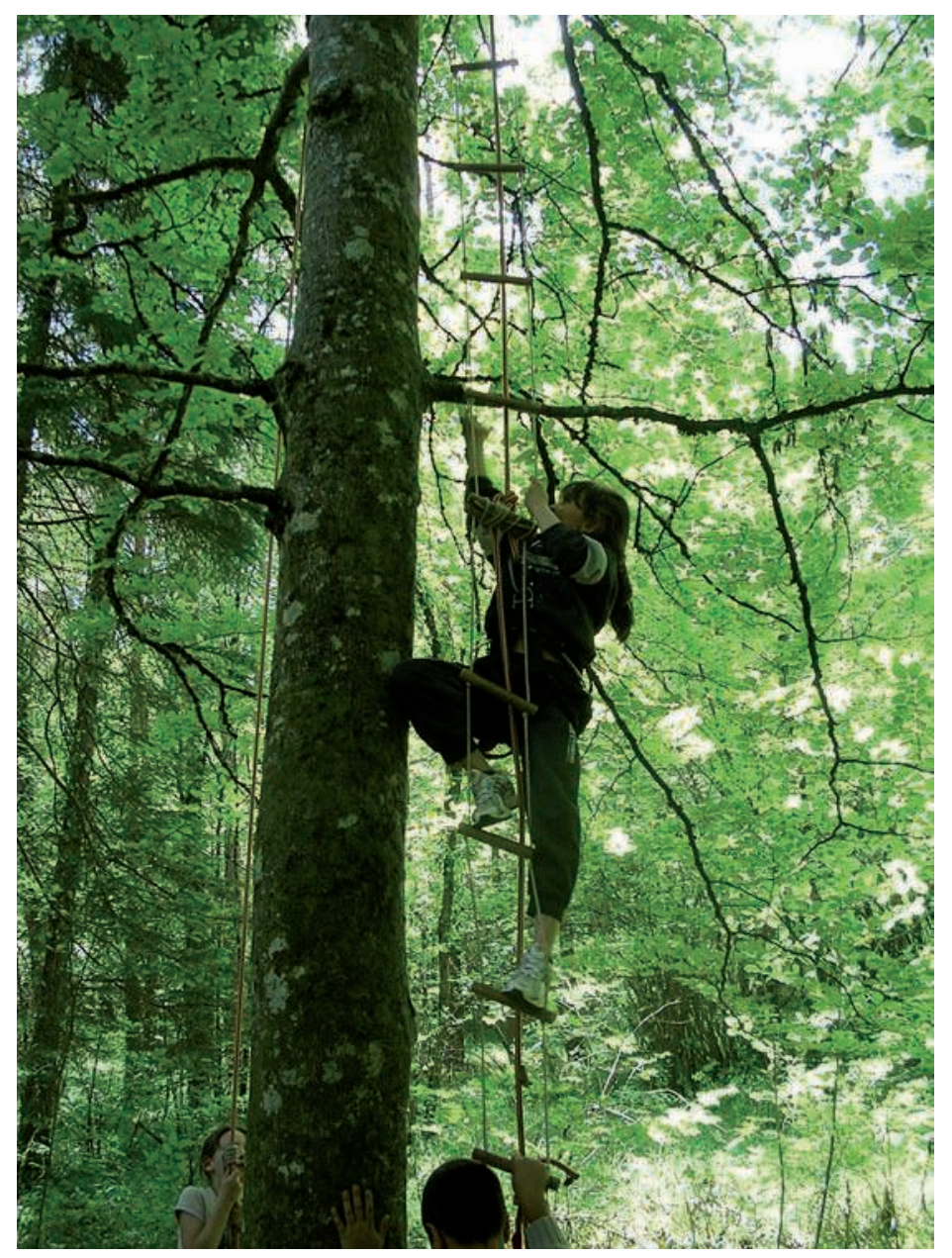

Activités d'accrobranches proposées par les animateurs de la maison de la Forêt pour sensibiliser « corporellement» les visiteurs. (c) Maison de la Forêt 
largement est proposée une sensibilisation au thème et des réponses aux questions que se posent les participants ou quilis seront appelés à se poser dans leur future vie professionnelle.

Lultime catégorie de public, également prise en compte lors de la conception de l'exposition, est celle du public de passage. Pour le développement du tourisme vert en milieu rural, la maison de la Forêt est un outil pour l'accueil dans la région. Elle participe à enrichir l'offre et à valoriser ce qui représente l'attrait et la particularité de la région, autrement dit la forêt. Le propos de l'exposition prend à contre-pied le visiteur qui n'en a pas une bonne connaissance, il repose sur l'idée que la forêt est un espace culturel plus qu'un espace naturel. En affirmant que l'homme a toujours modelé la forêt, que les activités qui s'y déroulent sont multiples, que s'y côtoient des intérêts divergents et que le tout repose sur une gestion équilibrée de ces activités, le lieu se veut aussi un espace de négociation entre des points de vue divergents. L'exposition est donc un lieu de sensibilisation pour prévenir des comportements et éduquer au respect de l'écosystème forestier.

Enfin, d'autres actions sont développées auprès des randonneurs, des amateurs de parcours d'aventures avec des activités de plein air, auprès des amis de la nature avec des conseils sur les champignons, ou des informations sur la faune sauvage, les truffes, l'écoute du brame du cerf... La population locale n'est évidemment pas oubliée, d'abord parce qu'elle est souvent partie prenante du bon fonctionnement du lieu, mais aussi parce qu'elle répond présente pour des rendez-vous plus festifs, tel que la fête de l'automne... Des partenariats privilégiés sont conduits également avec les entreprises et les acteurs de la filière bois (COFOR 21, Communes Forestières de Côte-d'Or, Union des entreprises du bois de Bourgogne...), mais aussi des associations de protection de la nature (Ligue de Protection des Oiseaux, Conservatoire des Sites Naturels Bourguignons...) afin de proposer des journées naturalistes et des approches thématiques renouvelées du milieu. L'intégration prochaine espérée au réseau des CPIE (Centres permanents d'initiatives pour l'environnement) va dans ce sens.

Ce site nous semble emblématique de ce à quoi doit correspondre un centre d'interprétation : sensibilisation, approche multidisciplinaire, combinaison des techniques, incitation faite aux visiteurs à s'interroger, carrefour d'approches dynamiques sur un même thème. Demeure le point crucial et encore mal résolu de ses moyens de fonctionnement. Si les partenaires économiques ont contribué à sa genèse, ils participent encore modestement à son fonctionnement, de même que les différents niveaux des collectivités territoriales. Si bien que le lieu est bridé dans son développement par manque de moyens et de compétences internes, et que par conséquent le risque est grand de voir se dégrader lentement un équipement pertinent, mais qui n'aura pas eu l'occasion de se déployer pleinement. Le cas n'est pas exceptionnel, il est même assez représentatif d'une multitude de lieux, inaugurés avec enthousiasme puis abandonnés à leur quotidien difficile. 